Faculdade

de Ciências Econômicas UFRGS
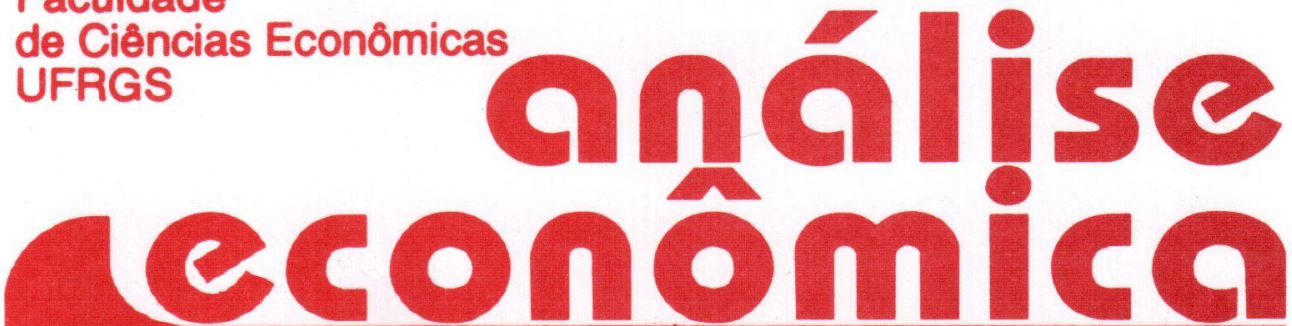

- A teoria NEOCLÁssica (PURA) E A TEORIA NEO-AUSTRIACA FRENTE AO LEGADO CARTESIANO Eleutério F. S. Prado

- MEdidAs de CONCENTRAÇÃo INDUSTRIAL: UMA RESENHA Marcelo Resende

- SISTEMA TRIBUTÁRIO E IMPOSTO ÚNICO SOBRE TRANSAÇÕES Ricardo Letizia Garcia

- ECONOMIA DO NORDESTE: TENDÊNCIAS DAS ÁREAS DINÂMICAS Policarpo Lima

- Custos e BENEFÍcIos DA INTEGRAÇÃO DO GRUPO ANDINO Marco Antônio Montoya

- Pós-graduAÇÃo EM ECONOMIa NOS ESTADOS UNIDOS DA AMÉRICA Egon Roque Fröhlich

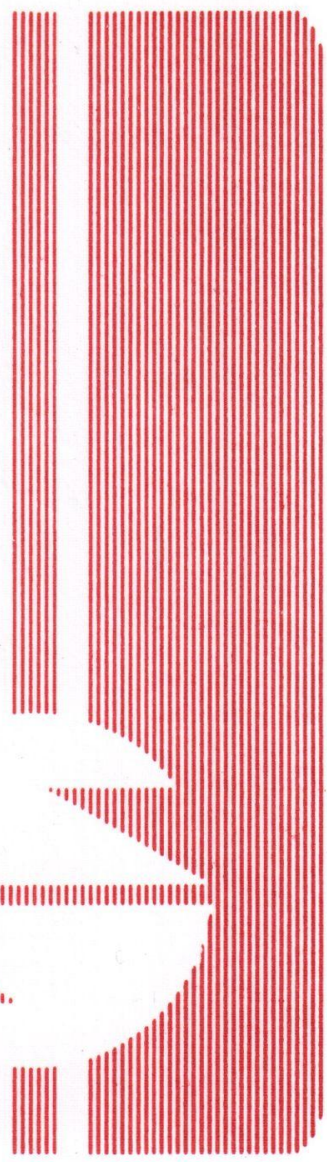


UNIVERSIDADE FEDERAL DO RIO GRANDE DO SUL

Reitor Prof Hélgio Henrique Casses Trindade

FACULDADE DE CIÊNCIAS ECONÓMICAS

Diretor Prof Pedro Cézar Dutra Fonseca

CENTRO DE ESTUDOS E PEQUISAS ECONÔMICAS

Diretor Prof. Paulo Alexandre Spohr

DEPARTAMENTO DE CIÉNCIAS ECONÓMICAS

Chefe: Prof Achyles Barcelos da Costa

CURSO DE PÓS-GRADUAÇÃO EM ECONOMIA

Coordenador Prof Nali de Jesus de Souza

CURSO DE PÓS-GRADUAÇÃO EM ECONOMIA RURAL

Coordenador. Prof Atos Freitas Grawunder

CONSELHO EDITORIAL: Achyles Barcelos da Costa, Aray Miguel Feldens, Atos Freitas Grawunder, Carlos Augusto Crusius, Fernando Ferrari Filho, Juvir Luiz Mattuella, Marcelo Savino Portugal, Maria Imilda da Costa e Silva, Nali de Jesus de Souza, Nuno R. L. de Figueiredo Pinto, Otília Beatriz K. Carrion, Paulo Alexandre Spohr, Pedro Cezar Dutra Fonseca, Roberto Camps Moraes, Valter José Stülp, David Garlow (Wharton Econometrics Forecasts Association, E.U.A.), Edgar Augusto Lanzer (UFSC), Eleutério Prado (USP), Fernando de Holanda Barbosa (FGV/RJ), Gustavo Franco (PUC/RJ), João Rogério Sanson (UFSC), Joaquim Pinto de Andrade (UnB), Juan H. Moldau (USP), Werner Baer (Univ. de Illinois, E.U.A.).

COMISSÃO EDITORIAL: Atos Freitas Grawunder, Pedro Cezar Dutra Fonseca, Marcelo Savino Portugal, Roberto Camps Moraes.

EDITOR: Nali de Jesus de Souza

SECRETARIA Cláudia Porto Silveira, Jeferson Luis Bittencourt e Vanete Ricachescki (revisão de textos)

FUNDADOR: Prof Antônio Carlos Santos Rosa

Os materiais publicados na revista Análise Econômica são da exclusiva responsabilidade dos autores. É permitida a reprodução total ou parcial dos trabalhos, desde que seia citada a fonte. Aceita-se permuta com revistas congêneres. Aceitam-se, também, livros para divulgação, elaboração de resenhas e recensōes. Toda correspondência, material para publicação (vide normas na terceira capa), assinaturas e permutas devem ser dirigidos ao seguinte destinatário:

PROF. NALI DE JESUS DE SOUZA

Revista Análise Econômica

Av. João Pessoa, 52

CEP 90040-000 PORTO ALEGRE - RS, BRASIL

E-MAIL: NALI@VORTEXUFRGS.BR

Telefones: (051) 316-3348 e 316-3440

Fax: (051) 225-1067 


\section{DISSERTAÇÓES DE MESTRADO DE ECONOMIA \\ E ECONOMIA RURAL DA UFRGS, DEFENDIDAS EM 1994}

\section{ECONOMIA}

FLORES, Luciano Lauri. As Exportaçסees Brasileiras de Manufaturados e o Novo Protecionismo nos Países Desenvolvidos. (Orientador: Prof. Roberto Camps de Moraes).

GARCIA, Ricardo Letizia. O Imposto Único sobre Transações e a sua Repercussão na Economia. (Orientador: Prof. João Rogério Sanson).

GARAGORY, Jorge Alano Silveira. Indice de Sucesso dos Trabalhadores do Rio Grande do Sul em Negociaçőes Coletivas. (Orientador: Profa Maria Imilda da Costa e Silva).

JORGE, Isabel. Privatização Experiências Internacionais, Aspectos Teóricos e Resultados. (Orientador: Prof. Roberto Camps de Moraes).

MARQUES, Murilo Machado. Integração Económica Brasil-Argentina: Um Estudo Teórico e Empirico dos Efeitos Potenciais sobre o Fluxo de Comércio Bilateral (Orientador: Prof. Ernani Hickmann).

MOURA NETO, Bolivar Tarrago. Política Salaria e Salário-Real no Brasil: 19641990. (Orientador: Prof. Roberto Camps de Moraes).

SCHERER, André Luis Forti. A Estratégia Emnresarial e os Determinantes da Difusão de Tecnologia: Um Estudo sobre a Industria de Vestuário do Rio Grande do Sul. (Orientador. Prof. Achyles Barcelos da Costa)

VARGAS, Marco Antônio. Subcontratação e Inovação Tecnológica na Indústria Brasileira de Máquinas e Implementos Agicolas. (Orientador: Prof. Achyles Barcelos da Costa). 


\section{ECONOMIA RURAL}

ARBAGE, Alessandro Porporatti. Análise Económica do Transporte de Soja em Grão no Estado do Rio Grande do Sul (o Caso do Porto de Cachoeira do Sul). (Orientador: Prof. Aray Miguel Feldens).

AREND, Silvio Cezar. Análise da Viabilidade Econômica da Industrialização da Cana-de-Açúcar em Microdestilaria. (Orientadores: Profa. Nelci Maria Ritcher Giacomini e Prof. Reinaldo Ignácio Adams).

GOMES, Mário Conill. Avaliação Econômica da Conservação do Solo em Microbacias do Municipio de Marau - RS. (Orientador: Prof. Juvir Luiz Matuella).

GELINSKI Jr., Eduardo. Tipificação dos Sistemas Predominantes na Produção de Leite na Regiáo de Abrangéncia da Cooperativa Central de Laticínios do Paraná

- Microrregião Campos de Ponta Grossa e Jaguariaiva. (Orientador: Prof. Otto Gulherme Konzen).

FOCHEZATTO, Adelar. Análise da Carga Tributária Incidente sobre as Cadeias Agroindustriais do Arroz e Soja. (Orientador: Prof. Juvir Luiz Matuella). 